\title{
An Optimized Reduced Order Model Using Interpolation Method and Design of Controller
}

\author{
T. Narasimhulu ${ }^{1}$ and G. Raja Rao ${ }^{2}$ \\ ${ }^{1}$ Avanthi Institute of Engg. \& Tech., ${ }^{2}$ ANITS, Sangivalasa, Visakhapatnam \\ 1narsim.223@gmail.com,profgrr@gmail.com
}

\begin{abstract}
The authors proposed a method for order reduction of linear dynamic systems using the advantages of the Interpolation criterion and Routh method. The denominator polynomial of the reduced order model is determined by using the Routh method while the numerator coefficients are computed by minimizing the integral square error between the original and the reduced system using Interpolation method. The proposed method guarantees stability of the reduced model, if the original high order system is stable system. A PID controller is designed for the high order original systems through its low order model proposed. Some numerical examples were considered to explain the effectiveness of the method.
\end{abstract}

Keywords: Interpolation criterion; Routh method; Stability; integral square error.

\section{Introduction}

The approximation of linear systems plays an important role in many engineering applications, especially in control system design, where the engineer is faced with controlling a physical system for which an analytic model is represented as a high order linear system.

In many practical situations a fairly complex and high order system is not only tedious but also not cost effective for on-line implementation. It is therefore desirable that a high system be replaced by a low order system such that it retains the main qualitative properties of the original system.

Several order reduction techniques for linear dynamic systems in the frequency domain are available the literature [1-4]. Further, some methods have also been suggested by combining the features of two different methods [5-7]. To overcome these problems model order reduction techniques are implemented. It is desirable to reduce higher order transfer function to low order model which are expected to approximate the performance of original high order system.

A mixed method is proposed for the reduction of high order continuous systems. This method is developed from the method [1] available in literature and it overcomes the limitations and drawbacks of some existing methods. In the present paper, Interpolation method is used for obtaining the numerator and Routh method is used for obtaining the denominator of the reduced order model.

Then an optimum model (with minimum ISE) is obtained by varying the interpolation points. Using the proposed method a PID controller is designed for the high order original systems.

\section{Stability \& Choice of The Interpolation Points}

Because the above method cannot ensure the reduction model stable, it is required to choose its denominator such that the reduction model is stable. In order to produce the dominator polynomial, the following two famous methods:

The retaining dominant poles and the Routh method [1, 3, and 5] will be introduced. Then its numerator can be produced by using the above method, and at this time, the number of points is $\partial(\hat{p})+1$.

Received: Desember 25 ${ }^{\text {th }}, 2012$. Accepted: May 31 ${ }^{\text {st }}, 2013$ 
By the way, the above method is similar to the classical Pade approximation when all the interpolation points are zeros; it only takes advantage of information of G(s) at zero. Usually, interpolation points chosen had beer reflect the features of the original model $G(s)$ well According to experience, we can choose the points which are located in the disk centered at the origin with radius: the distance between origin and the furthest poles; or besides some dominant poles; or besides origin.

\section{Reduction Procedure}

Let the transfer function of a higher order system be represented by [6], [7]

$$
G(s)=\frac{d_{0}+d_{1} s+\ldots+d_{n-1} s^{n-1}}{e_{0}+e_{1} s+\ldots+e_{n} s^{n}}=\frac{d_{n}(s)}{e_{n}(s)}
$$

Where $d_{i}, i=0,1 \ldots n-1$ and $e_{i}, i=0,1 \ldots n$ are constants.

For the high order system a reduced $\mathrm{k}^{\text {th }}$ order model is proposed as given below,

$$
R(s)=\frac{a_{0}+a_{1} s+\ldots+a_{k-1} s^{k-1}}{b_{0}+b_{1} s+\ldots+b_{k-1} s^{k-1}+b_{k} s^{k}}=\frac{a_{k}(s)}{b_{k}(s)}
$$

Where the $a_{i}, i=0,1 \ldots k-1$ and $b_{i}, i=0,1 \ldots k$ are constants.

\section{Reduced order denominator:}

Step 1: The denominator $\mathrm{b}_{k}$ (s) of reduced model can be obtained from the Routh Stability array of the denominator of the original system as given below:

The Routh array is formed by using the eqn. (4):

The Routh table for the denominator of the system is given below:

$$
\begin{aligned}
& b_{11}=e_{n} \quad b_{12}=e_{n-2} \quad b_{13}=e_{n-4} \quad b_{14}=e_{n-6} \cdots \\
& b_{21}=e_{n-1} b_{22}=e_{n-3} \quad b_{23}=e_{n-5} \quad b_{24}=e_{n-7} \cdots \\
& b_{31} \quad b_{32} \quad b_{33} \quad \ldots . . \\
& \text {..... } \\
& b_{k-1,1} \quad b_{k-1,2} \\
& b_{k, 1} \\
& b_{k+1,1} \\
& \text { Where } b_{i, j}=b_{i-2, j+1}-\frac{b_{i-2,1} b_{i-1, j+1}}{b_{i-1,1}} \text {, } \\
& \text { wher } \dot{\boldsymbol{\theta}} \geq \text { 3and } 1 \leq j \leq\left[\frac{(k-i+3)}{2}\right]
\end{aligned}
$$

$\mathrm{b}_{k}(\mathrm{~S})$ may be easily constructed from the $(\mathrm{n}+1-\mathrm{k})^{\text {th }}$ and $(\mathrm{n}+2-\mathrm{k}){ }^{\text {th }}$ and $(\mathrm{n}+2-\mathrm{k})^{\mathrm{th}}$ rows of the above to give

$$
b_{k}(s)=b_{k+1-n .1} s^{n}+b_{k+2-n .1} s^{n-1}+b_{k+1-n .2} s^{n-2}+\ldots
$$




\section{Reduced order numerator:}

The reduced order numerator polynomial is derived in the following sequence:

1) Choose $2 k$ point's $s_{0}, s_{1} \ldots s_{2 k-1}, s_{2 k} \in{ }_{C}$ (they can be multiple) from the location of the poles of the original systems and obtain $g(s)$ as given below:

$$
\begin{aligned}
g(s) & =\left(s-s_{0}\right)\left(s-s_{1}\right) \ldots\left(s-s_{2 k-1}\right) \\
& =\left(s-s_{0}\right)^{k_{0}}\left(s-s_{1}\right)^{k_{1}} \ldots .\left(s-s_{j}\right)^{k_{j}} \\
& =s^{2 k}+g_{2 k-1} s^{2 k-1}+\ldots .+g_{1} s+g_{0}
\end{aligned}
$$

2) Divide $d_{n}(s) b_{k}(s)$ by g(s) to get the quotient e(s) and the remainder $\mathrm{f}(\mathrm{s})$.

3) Divide $e_{n}(s) a_{k}(s)$ by g(s) to get the quotient $\mathrm{l}(\mathrm{s})$ and the remainder $\mathrm{h}(\mathrm{s})$. It yields,

$$
\begin{aligned}
& d_{n}(s) b_{k}(s)=g(s) e(s)+f(s) \\
& e_{n}(s) a_{k}(s)=g(s) l(s)+h(s)
\end{aligned}
$$

Where both $f(s)$ and $h(s)$ are polynomials of degree at most (2k-1),

$$
\begin{aligned}
& \left.\left(d_{n}(s) b_{k}(s)\right)^{(k)}\right|_{s i}=\left.f^{(k)}\right|_{s i}=\left.\left(e_{n}(s) a_{k}(s)\right)^{(k)}\right|_{s i}=\left.h^{(k)}\right|_{s i} \\
& i=0,1, \ldots, j, \quad k=0,1, \ldots, k_{i-1} \cdot \sum_{i=0}^{j} k_{i}=2 k
\end{aligned}
$$

By using the basic theorem of algebra, it is obtained that [1],

$$
f(s) \equiv h(s)
$$

It is found that the coefficient of each term in $\mathrm{f}(\mathrm{s})$ is the linear combination of $a_{0}, a_{1} \ldots, a_{2 k-1}$ and the coefficient of each term in $\mathrm{h}(\mathrm{s})$ is the linear combination of $b_{0}, b_{1} \ldots, b_{2 K-1}$. A linear system with $2 \mathrm{k}+1$ unknowns and $2 \mathrm{k}$ equations is formed. Hence, $b_{k}$ is assumed as ' 1 ' to make the no. of equations equal to the no. of unknowns.

If the coefficient matrix of the above linear system is non-singular, then its solution $b_{0}, b_{1} \ldots, b_{k}, a_{1} \ldots, a_{k-1}$ can be uniquely determined by using the Crammers rule.

\section{Numerator reduction procedure:}

Step 1: Choose $2 \mathrm{k}$ point's $\mathrm{s}_{0}, \mathrm{~s}_{1} \ldots \mathrm{s}_{2 \mathrm{k}-1}, \mathrm{~s}_{2 \mathrm{k}} \in \mathrm{c}_{\mathrm{c}}$ (they can be multiple) from the location of the poles of the original systems and obtain $\mathrm{g}(\mathrm{s})$ as given below:

$$
\begin{aligned}
g(s) & =\left(s-s_{0}\right)\left(s-s_{1}\right) \ldots .\left(s-s_{2 k-1}\right) \\
& =\left(s-s_{0}\right)^{k_{0}}\left(s-s_{1}\right)^{k_{1}} \ldots .\left(s-s_{j}\right)^{k_{j}} \\
& =s^{2 k}+g_{2 k-1} s^{2 k-1}+\ldots .+g_{1} s+g_{0}
\end{aligned}
$$

Step 2: Compute $\mathrm{d}_{\mathrm{n}}(\mathrm{s}) \mathrm{b}_{\mathrm{k}}(\mathrm{s})$ and $\mathrm{e}_{\mathrm{n}}(\mathrm{s}) \mathrm{a}_{\mathrm{k}}(\mathrm{s})$, respectively, 
T. Narasimhulu, et al.

$$
\begin{aligned}
& d_{n}(s) b_{k}(s)=\left(d_{n-1} s^{n-1}+\ldots+d_{0}\right)\left(b_{k} s^{k}+\ldots+b_{0}\right) \\
& \quad=c_{k+n-1}^{(0)} s^{k+n-1}+\ldots .+c_{1}^{(0)} s+c_{0}^{(0)} \\
& c_{0}^{(0)}=b_{0} d_{0}, \\
& c_{1}^{(0)}=b_{0} d_{1}+b_{1} d_{0}, \\
& \ldots . \\
& c_{k+n-2}^{(0)}=b_{k} d_{n-2}+b_{k-1} d_{n-1}, \\
& c_{k+n-1}^{(0)}=b_{k} d_{n-1,} \\
& a n d \quad \\
& e_{n}(s) a_{k}(s)=\left(e_{n} s^{n}+\ldots+e_{0}\right)\left(a_{k-1} s^{k-1}+\ldots+a_{0}\right) \\
& \quad=d_{k+n-1}^{(0)} s^{k+n-1}+d_{k+n-2}^{(0)} s^{k+n-2}+\ldots \\
& \quad+d_{1}^{(0)} s+d_{0}^{(0)}, \\
& d_{0}^{0}=e_{0} a_{0} \\
& d_{1}^{(0)}=a_{0} e_{1}+a_{1} e_{0}, \\
& \ldots . \\
& d_{k+n-2}^{(0)}=a_{k} e_{n-1}+a_{k-2} e_{n,}, \\
& d_{k+n-1}^{(0)}=a_{k-1} e_{n} .
\end{aligned}
$$

Step 3: Divide $d_{n}(s) b_{k}(s)$ by g (s) to get $\mathrm{f}(\mathrm{s})$ :

$$
\begin{aligned}
& c_{k+m}^{(0)} s^{n k-1}+c_{k+n}^{(1)} s^{n k 2}+\ldots \\
& s^{2 k}+g_{2 k+1} s^{2 k-1}+\ldots+g_{s} s+g_{0} \sqrt{c_{k+n 1}^{(0)} s^{k+n 1}+c_{k+n 2}^{(0)} s^{k+n 2}+\ldots+c_{n k 1}^{(0)} s^{n k 1}+\ldots+c_{0}^{(0)}} \\
& \frac{c_{k+m 1}^{(0)} s^{k+n-1}+g_{k-1} c_{k+n 1}^{(0)} s^{k+n 2}+\ldots+g_{0} c_{k+n 1}^{(0)} s^{n k 1}}{c_{k+n 2}^{(D)} 2^{k+n 2}+\ldots+c_{n k 2}^{(D)} 2^{n k 3}+\ldots+c_{0}^{(D)}} \\
& \frac{c_{k+m}^{(1)} 2^{k+n 2}+. .+g_{b} k_{k m+n}^{(1)} 2^{n k 2}}{c_{k+n}^{(2)} 3^{k+n 3}+. .+c_{0}^{(2)}}
\end{aligned}
$$

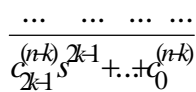

Thus get the recursive relations 


$$
\begin{gathered}
c_{i}^{(1)}=c_{i}^{(0)}-c_{k+n-1}^{(0)} g_{i+k-n+1,} \\
i=0,1, \ldots, k+n-2, \\
c_{i}^{(2)}=c_{i}^{(1)}-c_{k+n-2}^{(1)} g_{i+k-n+2,} \\
i=0,1, \ldots, k+n-3, \\
\text { relations: } c_{i}^{(3)}=c_{i}^{(2)}-c_{k+n-3}^{(2)} g_{i+k-n+3,} \\
i=0,1, \ldots, k+n-4, \\
\ldots \ldots . \\
c_{i}^{(l)}=c_{i}^{(l-1)}-c_{k+n-l}^{(l-1)} g_{i+k-n+l}, \\
c_{i}^{(n-k)}=c_{i}^{(n-k-1)}-c_{2 k}^{(n-k-1)} g_{i,}, \\
i=0,1, \ldots, 2 k-1,
\end{gathered}
$$

Divide $e_{n}(s) a_{k}(s)$ by g (s) to get h(s):

$$
\begin{aligned}
& d_{k+n}^{(0)} s^{n k-1}+d_{k+n-2}^{(1)} 2^{n k-2}+\ldots \\
& s^{2 k}+g_{2 k-1} s^{2 k-1}+\ldots+g_{1} s+g_{0} \sqrt{d_{k+n}^{(0)} s^{k+n-1}+d_{k+n-2}^{(0)} s^{k+n-2}+\ldots+d_{n k-1}^{(0)} s^{n k-1}+\ldots+d_{0}^{(0)}} \\
& \frac{d_{k+1}^{(0)} s^{k+n-1}+g_{2 k-1} d_{k+n}^{(0)} s^{k+n-2}+\ldots+g_{0} d_{k+1}^{(0)} 1^{n-k-1}}{d_{k+n-2}^{(1)} s^{k+n-2}+\ldots+d_{n k-2}^{(1)} s^{n k-3}+\ldots+d_{0}^{(1)}} \\
& \frac{d_{k+n-2}^{(1)} 2^{k+n-2}+\ldots+g_{0} d_{k+n}^{(1)} 2^{n-k-2}}{d_{k+n-3}^{(2)} 3^{k+n-3}+\ldots+d_{0}^{(2)}} \\
& \begin{array}{llll}
\ldots & \ldots & \ldots & \ldots \\
d_{2 k-1}^{(n-k)} s^{2 k-1}+\ldots+d_{0}^{(n-k)} &
\end{array}
\end{aligned}
$$

Thus get the recursive relations:

$$
\begin{aligned}
& d_{i}{ }^{(1)}=d_{i}{ }^{(0)}-d_{k+n-1}^{(0)} g_{i+k}-n+1, \\
& i=0,1, \ldots, \quad k+n-2, \\
& d_{i}^{(2)}=d_{i}^{(1)}-d_{k+n-2}^{(1)} g_{i+k}-n+2 \text {, } \\
& i=0,1, \ldots, \quad k+n-3, \\
& d_{i}^{(3)}=d_{i}^{(2)}-d_{k+n-3}^{(2)} g_{i+k}{ }^{(2)}-n+3 \text {, } \\
& i=0,1, \ldots, k+n-4, \\
& \text { ….... } \\
& d_{i}{ }_{i}^{(l)}=d_{i}^{(l-1)}-d_{k+n-1}^{(l-1)} g_{i+k-n+l}, \\
& d_{i}^{(n-k)}=d_{i}^{(n-k-1)}-d_{2 k}^{(n-k-1)} g_{i} \text {, } \\
& i=0,1, \ldots, 2 k-1 \text {, }
\end{aligned}
$$

When $k<0$, let $g_{o}=0$. In the above the recursive relations, the superscript $\mathrm{n}$ in $C_{i}^{(n)}$ represent the coefficients which are obtained after carrying out the algorithm $n$ steps. And the subscript 'i' in $C_{i}^{(n)}$ represents the corresponding degree about the variables.

Step 4: By using the basic theorem of algebra, it is obtained that [1], [10]

$$
f(s) \equiv h(s) .
$$

It is found that the coefficient of each term in $f(s)$ in is the linear combination of $a_{0}, a_{1} \ldots, a_{2 k-1}$ and the coefficient of each term in $h(s)$ is the linear combination of $b_{0}, b_{1} \ldots, b_{2 K-1}$. 
A linear system with $2 k+1$ unknowns and $2 k$ equations is formed assuming $b_{k}=1$. Thus, the reduced model is obtained as given equation (2).

\section{Optimized Reduced Order Model (OROM)}

Steps to obtain an optimum ROM:

$>$ Obtain reduced order denominator by using Routh method.

$>$ Obtain reduced order numerator by using Interpolation method.

$>$ Calculate ISE (Integral Square error)

$>$ Change the set of Interpolation points randomly for obtaining another reduced order numerator.

$>$ Again calculate ISE (Integral Square error) with the new numerator.

$>$ Repeat the above steps with different sets of interpolation points.

$>$ Pick the minimum ISE of all and the corresponding reduced order model is selected as an optimized model.

\section{Advantages of The Proposed Method}

The following are the advantages of the proposed method:

$>$ This method always gives stable reduced order models for the stable original Systems.

$>$ This method gives zero steady state error.

$>$ This method is simple and efficient.

$>$ This method gives very low ISE comparing to some other existing methods. So, reduced order model is better approximation of the original system.

$>$ This method retains both initial time moments and Markova parameters.

\section{Numerical Examples}

Example 1: consider the sixth order system as given

$$
G_{6}(s)=\frac{s^{4}+13 s^{3}+63 s^{2}+133 s+102}{s^{6}+14.5 s^{5}+81 s^{4}+223 s^{3}+318 s^{2}+212.5 s+50}=\frac{d_{n}(s)}{e_{n}(s)}
$$

A Second order reduced model is obtained for the above higher order system, in following steps, using the proposed method given in section-3.

$$
R_{2}(s)=\frac{a_{0}+a_{1} s}{b_{0}+b_{1} s+b_{2} s^{2}}=\frac{a_{k}(s)}{b_{k}(s)}
$$

Step 1: Reduced Order denominator is obtained by using Routh method as below:

$$
e_{n}(s)=s^{6}+14.5 s^{5}+81 s^{4}+223 s^{3}+318^{2}+2125 s+50
$$

\section{Routh Table:}

$\begin{array}{ccccc}s^{6} & 1 & 81 & 318 & 50 \\ s^{5} & 14.5 & 223 & 212.5 & \\ s^{4} & 65.62 & 303.3 & 50 & \\ s^{3} & 155.9 & 201.2 & & \\ s^{2} & 218.6 & 50 & & \\ s^{1} & 165.5 & & & \\ s^{0} & 50 & & & \end{array}$

Hence, the reduced order denominator is:

$b_{k}(s)=218.6 s^{2}+165.5 s+50$ 
By normalizing the above, the reduced order denominator is:

$b_{k}(s)=s^{2}+0.7574 s+0.2288$

Step 2: The numerator of reduced order model is obtained by the interpolation method as given in proposed procedure.

For a $2^{\text {nd }}$ order model the 4 required interpolation points are selected randomly as:

$0,0.6,2,3$

$g(s)=s^{4}-5.6 s^{3}+9 s^{2}-3.6 s$

Where $\mathrm{g}(\mathrm{s})$ is the polynomial obtained by the selected interpolation points.

From original order numerator and reduced order denominator,

$$
\begin{aligned}
& d_{n}(s) b_{k}(s)=\left(s^{4}+13 s^{3}+63 s^{2}+133 s+102\right)\left(s^{2}+0.7574 s+0.2288\right) \\
& d_{n}(s) b_{k}(s)=4.372 s^{6}+60.15 s^{5}+319.5 s^{4}+803 s^{3}+949.2 s^{2}+470.6 s+102
\end{aligned}
$$

Divide $d_{n}(s) b_{k}(s)$ by g (s) to get the quotient e(s) and the remainder $\mathrm{f}(\mathrm{s})$. Thus,

$$
f(s)=4276.42 s^{3}-5523.93 s^{2}+3181.04 s+102
$$

From original order numerator and reduced order denominator,

$$
\begin{aligned}
& e_{n} a_{k}=\left(s^{6}+145 s^{5}+81 s^{4}+223^{3}+318^{2}+2125 s+50\right)\left(a_{1} s+a_{0}\right) \\
& e_{n} a_{k}=a_{1} s^{7}+\left(14.5 a_{1}+a_{0}\right) s^{6}+\left(81 a_{1}+14.5 a_{0}\right) s^{5} \\
& +\left(223 a_{1}+81 a_{0}\right) s^{4}+\left(318 a_{1}+223 a_{0}\right) s^{3} \\
& \quad+\left(2125 a_{1}+318 a_{0}\right) s^{2}+\left(50 a_{1}+21.5 a_{0}\right) s+50 a_{0}
\end{aligned}
$$

Divide $e_{n} a_{k}$ by g(s) to get the quotient l(s) and the remainder h(s).

Thus,

$$
\begin{aligned}
& h(s)=\left(107923 a_{0}+4773 a_{1}\right) s^{3}+\left(127068 a_{0}+82961 a_{1}\right) s^{2} \\
& +\left(876.91 a_{0}+3935228 a_{1}\right) s+50 a_{0}
\end{aligned}
$$

From equn (1) \& equn (2), we get

$$
\begin{aligned}
& a_{0}=0.0809 \\
& a_{1}=0.466
\end{aligned}
$$

Hence, the reduced model is:

$$
R_{2}(s)=\frac{0.0809 s+0.466}{s^{2}+0.75838 s+0.2287}
$$


T. Narasimhulu, et al.

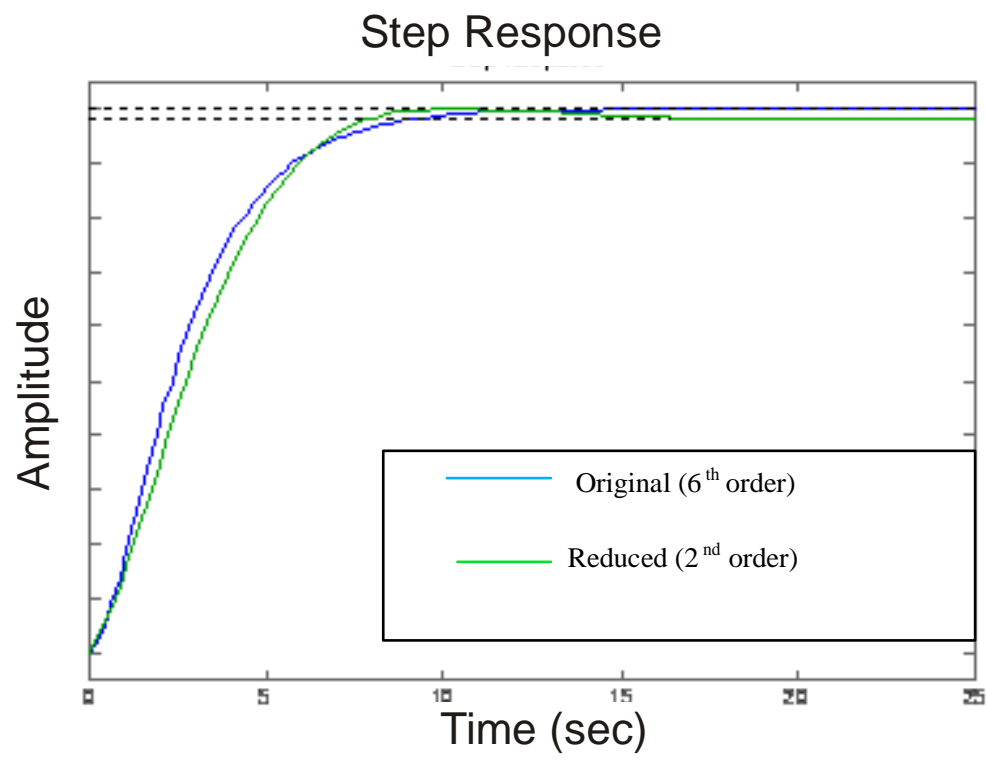

Figure 1. Comparison of step response of original and

\begin{tabular}{|c|c|c|c|}
\hline Original Order system & $\begin{array}{c}\text { Interpolatio } \\
\text { n points }\end{array}$ & Reduced order system & ISE \\
\hline$G(s)=\frac{s^{4}+13^{3}+63^{2}+133+102}{s^{6}+145 s^{5}+8 s^{4}+223^{3}+318^{2}+215 s+50}$ & $\begin{array}{l}0,- \\
0.5,0.9+1.78 \\
i, 0.9-1.78 \mathrm{i}\end{array}$ & $R_{2}(s)=\frac{1.34 s+0.46629}{s^{2}+0.75838+0.2287}$ & 0.186 \\
\hline$G(s)=\frac{s^{4}+133^{3}+63^{2}+133+102}{s^{6}+145 s^{5}+81 s^{4}+223^{3}+318^{2}+2125 s}$ & $0,0.6,2,3$ & $R_{2}(s)=\frac{0.080 \$+0.466}{s^{2}+0.75838+0.2287}$ & 0.148 \\
\hline$G(s)=\frac{s^{4}+13 s^{3}+63 s^{2}+133+102}{s^{6}+145 s^{5}+81 s^{4}+223^{3}+318^{2}+2125 s}$ & $\begin{array}{l}0,-3.5289 \\
0.11+1.78 \mathrm{i} \\
0.11-1.78 \mathrm{i}\end{array}$ & $R_{2}(s)=\frac{0.261988+0.46629}{s^{2}+0.75838+0.2287}$ & $\begin{array}{l}0.042 \\
34\end{array}$ \\
\hline
\end{tabular}

Example 2: Consider the $8^{\text {th }}$ order system as follows:

$$
\begin{aligned}
& \mathrm{G}(\mathrm{s})=\frac{d_{n}(s)}{e_{n}(s)} \\
& d_{n}(s)=0.01141 s^{7}+0.5119 s^{6}+2.4152 s^{5}+4.918 s^{4} \\
&+ 6.2164 s^{3}+4.6146 s^{2} \\
&+ 1.7134 s+0.261 \\
& e_{n}(s)=s^{8}+ 9.83 s^{7}+36.616 s^{6}+65.852 s^{5}+73.018 s^{4} \\
&+ 50.03 s^{3}+17.104 s^{2} \\
&+ 1.919 s+0.25
\end{aligned}
$$


A Second order reduced model is obtained for the above higher order system, in following steps, using the proposed method given in section-3.

$$
R_{2}(s)=\frac{a_{0}+a_{1} s}{b_{0}+b_{1} s+b_{2} s^{2}}=\frac{a_{k}(s)}{b_{k}(s)}
$$

By normalizing the above, the reduced order denominator is(by applying routh method): $b_{k}(s)=s^{2}+0.07881 s+0 . .0186$

Reduced order numerator (by applying Interpolation method):

Using the procedure the optimum reduced order model is obtained as,

$$
R_{2}(s)=\frac{0.05244 s+0.0194}{s^{2}+0.07881 s+0.0186}
$$

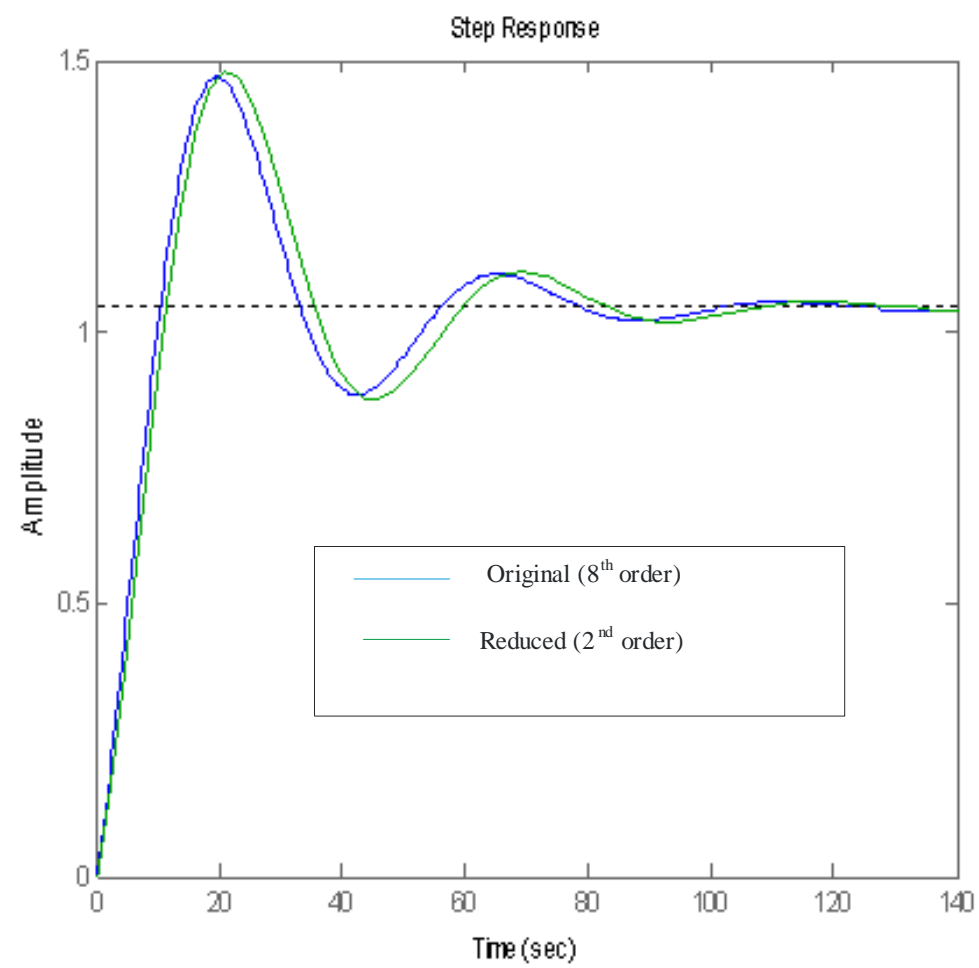

Figure 2. Step response of original and reduced order systems.

Design of PID controller for reduced order model is, Let transfer function of the PID controller is,

$$
G_{C}(s)=\frac{k_{d} s^{2}+k_{p} s+k_{i}}{s}
$$

Applying ITAE performance index method to the reduced model, the values of Kp, Ki and Kd are obtained 
T. Narasimhulu, et al.

$$
R_{2}(s)=\frac{0.05244+0.0194}{s^{2}+0.07881+0.0186}
$$

Now to obtain the closed-loop transfer function for reduced order

The tuned PID values are

$$
K_{p}=-9.1331, \quad K_{i}=7.654, \quad K_{d}=23.899
$$

Comparing characteristic equation of compensated system to the optimum ITAE characteristic equation as,

$$
s^{3}+1.75 w_{n} s^{2}+3.25 w_{n}^{2} s+w_{n}^{3}
$$

The PID controller is added to the forward path and the closed loop transfer function with unity feedback of the system is given as:

$$
T_{c}(s)=\frac{G_{C}(S) G(S)}{1+G_{C}(S) G(S)}
$$

Where $G(s)$ is the high order system and $G_{c}(s)$ is the PID controller transfer function.

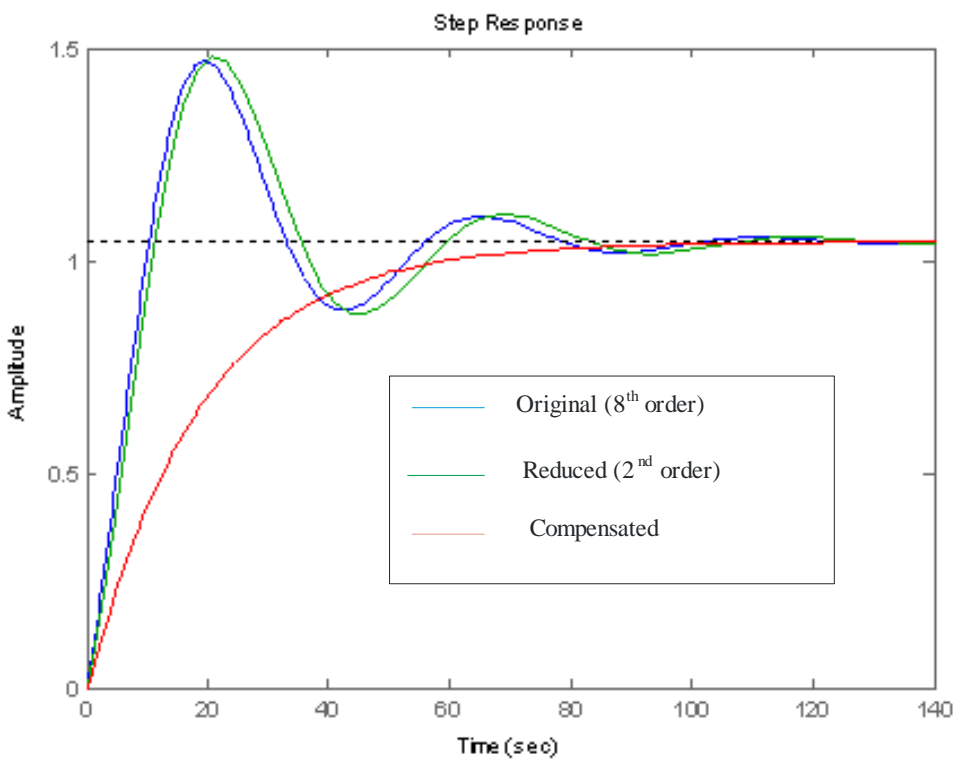

Figure 3. Step response of compensated system and uncompensated systems

Comparing With Existing Methods:

Example 3: Consider the $6^{\text {th }}$ order original transfer function is given by [13],

$$
G(s)=\frac{s^{5}+9 s^{4}+225 s^{3}+30.25 s^{2}+23.25 s+9}{s^{6}+10.5 s^{5}+45 s^{4}+100 s^{3}+119 s^{2}+69.5 s+15}
$$


The reduced second order models obtained by using proposed method is:

$$
R_{2}(s)=\frac{4.0761 s+3.1116}{s^{2}+0.670885 s+0.19281}
$$

The reduced $2^{\text {nd }}$ order models obtained by using Routh approximation method

$$
r_{2}(s)=\frac{0.465 s+0.09}{s^{2}+0.7 s+0.15}
$$

The reduced $2^{\text {nd }}$ order models obtained by using Continued fraction method $c_{2}(s)=\frac{1.96 s+0.732}{s^{2}+2.45 s+1.223}$

The step responses of the reduced models obtained by proposed method, routh approximation method $\left(\mathrm{r}_{2}\right)$ and continued fraction method $\left(\mathrm{c}_{2}\right)$ Figure 4.

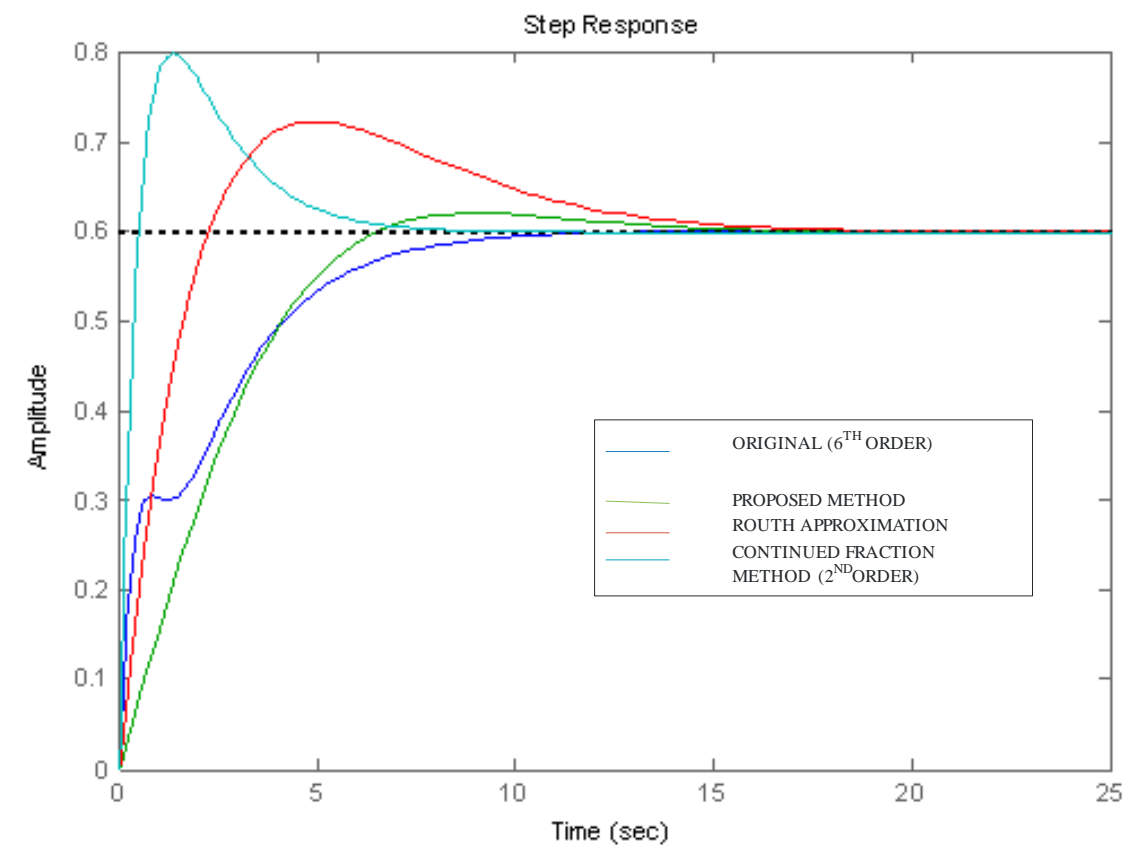

Figure 4. Step response of different existing methods 


\begin{tabular}{|l|l|l|}
\hline \multicolumn{1}{|c|}{ Original Order system } & \multicolumn{1}{|c|}{ Reduced order system } & ISE \\
\hline$G(s)=\frac{s^{5}+9 s^{4}+22.5 s^{3}+30.25 s^{2}+23.25 s+9}{s^{6}+10.5 s^{5}+45 s^{4}+100 s^{3}+119 s^{2}+69.5 s+15}$ & $\begin{array}{l}\text { c. f method } \\
c_{2}(s)=\frac{1.96 s+0.732}{s^{2}+2.45 s+1.223}\end{array}$ & 2.465 \\
\hline$G(s)=\frac{s^{5}+9 s^{4}+22.5 s^{3}+30.25 s^{2}+23.25 s+9}{s^{6}+10.5 s^{5}+45 s^{4}+100 s^{3}+119 s^{2}+69.5 s+15}$ & $\begin{array}{l}\text { R.A method } \\
r_{2}(s)=\frac{0.465 s+0.09}{s^{2}+0.7 s+0.15}\end{array}$ & 4.627 \\
\hline$G(s)=\frac{s^{5}+9 s^{4}+22.5 s^{3}+30.25 s^{2}+23.25 s+9}{s^{6}+10.5 s^{5}+45 s^{4}+100 s^{3}+119 s^{2}+69.5 s+15}$ & $\begin{array}{l}\text { Proposed method } \\
R_{2}(s)=\frac{4.0761 s+3.1116}{s^{2}+0.670885} s+0.19281\end{array}$ & 0.112 \\
\hline
\end{tabular}

\section{Conclusion}

In this paper, a new method is proposed for the reduction of high order continuous systems. The proposed method uses the application of Interpolation method for obtaining the numerator and Routh method for obtaining the denominator of the reduced order model. An optimum model (with minimum ISE) is derived by varying the interpolation points. Using the proposed method a PID controller is designed for the high order original systems. A computer program is developed for the proposed continuous systems reduction method.

\section{References}

[1] GU Chuan-quig, ZHANG Ying, "AnOsculatory rational interpolation method of model reduction in linear system”, Journal of Shanghai University (English Edition), 2007, 11(4):365-369...

[2] WU Bei-Bei, GU Chuan-quig. 'A matrix Pade Type Routh Model Reduction Method for Multivariable Linear Systems’ Journal of Shanghai University (English Edition), 2006, 10(5) : 377-380.

[3] Ismail O. On multipoint Pade approximation for discrete interval systems [C]//Proceedings of the Twenty Eighth Southeastern Symposium on System Theory Washington DC, USA: IEEE Computer Society, 1996: 497-501.

[4] Bandyopadhyay B, Ismail O, Gorez R. Routh-Padeapproximation for interval systems [J]. IEEE Transaction Automatic Control, 1994, 39: 2454-2456.

[5] Mohammad J. Large -scale Systems: Modeling and Control [M]. New York: Elsevier Science Publishing Press, 1983.

[6] Hutton M F, Friedland B. Routh approximations for reducing order of linear time invariant system [J]. IEEE Transaction Automatic Control, 1975, 20: 329-337.

[7] Baker G A, Jr, Graves - Morris P R. Pade Approximants [M]. 2nd ed. New York: Cambridge University Press, 1995.

[8] Gu Chuan-qing. Thiele-type and Lagrange-type generalized inverse rational interpolation for rectangular complex matrices [J]. Linear Algebra and Its Application, 1999, 295: 730 .

[9] J.V.B. Jyothi, G. Raja Rao, 'Approximation of Large Time Delay MIMO Systems by using Matrix Pade-Type Routh Model' , in proceedings of National Conference on CONTEMPORARY AND SOFTCOMPUTING in Electrical Engineering (ConCon2012) May 30-31. 
[10] Interpolation among reduced-order matrices to obtain parameterized models for design, optimization and probabilistic analysis, INTERNATIONAL JOURNAL FOR NUMERICAL METHODS IN FLUIDS Int. J. Number. Meth. Fluids 2010; 63:207-230 published online 8 June 2009 in Wiley InterScience (www.interscience.wiley.com). DOI: 10.1002/fld.2089.

[11] Parametric Model Order Reduction by Matrix Interpolation, at 8/ 2010, Heiko Panzer, Technische Universidad Munched, Jan Mohring, Fraunhofer Institute fur Techno- und Wirtschaftsmathematik, Kaiserslautern, Rudy Eid, Boris Lehmann, and Technische Universidad Munched.

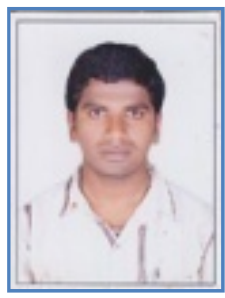

T. Narasimhulu obtained his post graduation in control systems from ANITS. He has published 5 papers so far.

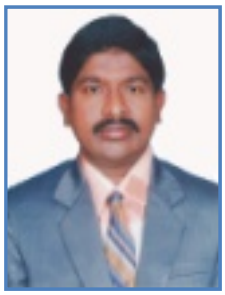

G. Raja Rao obtained his post graduation and Doctorate degrees from A. U. He has 23 years of teaching and industrial experience out of which more than 10 years experience as a professor. He has published more than 20 papers in National/ International journals/ conferences. His areas of research are Model order reduction, design of controller, non linear systems analysis \& design, etc... 